\title{
Diseño de un modelo de planificación de la mano de obra directa para la gestión de producción de empresas farmacéuticas
}

Gustavo Raúl QuisPe Canales ${ }^{1}$

RECIBIDO: 02/03/2018 ACEPTADO: 30/04/2019

\begin{abstract}
RESUMEN
En la industria farmacéutica, la planificación de la producción responde a las variaciones de la demanda de los clientes y de los procesos internos de la empresa; además, esta industria genera actividades que no agregan valor, sino más bien oportunidades de mejora como: a) las horas extra son mayor al $30 \%$, b) las reprogramaciones de la producción alcanzan el $40 \%$ y c) el nivel de servicio al cliente estatal alcanza el $70 \%$ de las órdenes de compra por atender. En ese sentido, la empresa tiene necesidad de mejorar esta situación, para lo cual se diseña un modelo de plan de ventas y operaciones donde se encuentra la planificación de la mano de obra directa (MOD), que va a permitir reducir las horas extra y el cálculo de la cantidad de MOD, en consideración de su nivel de eficiencia. En la práctica, este es el resultado esperado; su procedimiento se logra mediante las decisiones de la gerencia de operaciones.

Palabras-claves: Plan de producción; planificación de la MOD; capacidad de la MOD; método Kaizen; recurso crítico.
\end{abstract}

\section{INTRODUCCIÓN}

Al interpretar o definir el modelo S\&OP (Planeación de Ventas y Operaciones), Núñez et al. (2014) mencionan que:

El documento que recoge este nivel de planificación es el denominado plan agregado de producción, en cuyo diseño se tratará de igualar, siempre que sea posible, la tasa de producción a las previsiones de demanda. Sin embargo, dado que esta última suele no ser constante y regular a lo largo del tiempo, el planificador deberá decidir qué medidas de ajuste se deberán tomar para intentar igualarlas.

Para iniciar el diseño de un plan agregado, es pertinente conocer las necesidades de producción que se desean satisfacer. Estas se deben calcular a partir de las previsiones de demanda, considerando la existencia de inventarios disponibles (disminución de necesidades) o la existencia de pedidos pendientes de fabricar dejado por planificaciones anteriores (añadir necesidades). Así se obtiene el plan de producción, insumo principal para la planificación de la mano de obra directa.

De acuerdo a ello, Arnoletto (2007) comenta acerca de la secuencia de la planificación de los sistemas mrp (material requirement planning), la cual tiene como principal objetivo especificar cuál será la «combinación óptima de la tasa de producción, del nivel de la fuerza laboral y del inventario disponible». Asimismo, la tasa de producción está referida al «número de unidades terminadas por unidad de tiempo (como por hora o por día)»; mientras que el inventario disponible "es el saldo del inventario no utilizado traído desde el periodo anterior». Por último, «el nivel de la fuerza laboral es el número de trabajadores necesario para la producción» (p. 142).

La planificación de la mano de obra directa depende del modelo S\&OP, donde considera qué variables afectan al resultado. Con mayor profundidad es abordado por Peña (2017) en el siguiente análisis:

Magíster en Ingeniería Industrial por la Universidad Ricardo Palma. Actualmente, es docente y director de la Escuela Académico Profesional de Ingeniería Industrial de la URP. E-mail: gustavo.canales@urp.edu.pe 
En definitiva, el S\&OP trata de suavizar el efecto de las fluctuaciones de la demanda del mercado, así como los efectos de los demás cuellos de botella que pueden existir en la organización, y actúa como catalizador para evitar efectos bruscos que repercutan en la cuenta de resultados, y lograr un equilibrio final entre ventas y operaciones. (p. 84)

Los procesos de fabricación se ven afectados, adicionalmente, por las relaciones humanas y las variables del entorno, tales como los cambios económicos, sociales y políticos, y que afectan los resultados de los procesos empresariales; Joseph (2019) lo explica así:

Uncertainty can be measured by the frequency of its occurrence, and analyzing the relative contribution and resulting effect on delivery performance. It can quantify whether the impact is minor or major. Many conceptual and mathematical models are proposed and used to manage competitive production/manufacturing under uncertainties. Uncertainties in manufacturing have heterogeneous effects due to the interrelationships between resources and operations.

The lead-time and demand uncertainties are individually and interactively significant determinants of system performance found that as processing time uncertainty increases, simple dispatch heuristics provide performance comparable or superior to that of algorithmically more sophisticated scheduling policies. Again, increasing manufacturing flexibility leads to increased performance and to knob the uncertainty. (p. 679)

\section{PROBLEMA}

¿Cuál sería el impacto del diseño de un modelo de planificación de la mano de obra directa en la gestión de la producción de empresas farmacéuticas?

En el texto de Pacoricuna y Mejía (2015) se comenta sobre el sistema de producción de una empresa farmacéutica, y se afirma lo siguiente:

La tipología del sistema de producción corresponde a una producción por lotes con cuatro características principales. En primer lugar, el inicio de la fabricación es posible solo si se tiene la Orden de Trabajo, es decir, existe un pedido que solicita el producto para despacharlo al cliente. En segundo lugar, se cuenta con tres clientes denominados A, B y C. En tercer lugar, la variedad de productos está conformada por 40 diferentes productos; y por último, la demanda en el sector farmacéutico es fluctuante, lo que hace difícil obtener un pronóstico de fabricación en un periodo de tiempo mayor a 6 meses. (p. 2)

Las fluctuaciones de la demanda en el mercado farmacéutico son una variable frecuente; por lo tanto, es un factor importante a considerar con el fin de minimizar las desviaciones. Esto implica que debemos hacer un control y seguimiento de la planificación de la mano de obra directa, considerando la siguiente secuencia:

\section{PRONÓSTICO $\rightarrow$ PLANIFICACIÓN DE PRODUC-} CIÓN $\rightarrow$ PLANIFICACIÓN DE LA MOD

Donde la variable independiente es el pronóstico, la variable dependiente de primer nivel es la planificación de la producción y la variable dependiente de segundo nivel es la planificación de la mano de obra directa.

\section{PROBLEMAS ESPECÍFICOS}

¿Qué variables y parámetros logísticos y de producción intervienen en el proceso de la planificación de la MOD para lograr planes alternativos sobre su propia capacidad? ¿Cómo un modelo de planificación de MOD logra alternativas para minimizar los costos?

En su estudio, Heizer y Render (2008) mencionan cómo se integran la planificación del mediano plazo con el operativo:

Para los fabricantes, el plan agregado liga los objetivos estratégicos de la empresa con los planes de producción, pero en las empresas de servicios, el plan agregado relaciona los objetivos estratégicos con la programación de la mano de obra. (p. 111)

\section{METODOLOGÍA}

Del desarrollo del modelo de planificación de la mano de obra directa (MOD)

El objetivo es proporcionar la cantidad de personal de mano de obra directa que la empresa requiere; para ello, es importante considerar la responsabilidad social señalada por Fernández (2013):

La responsabilidad social no solo es aplicable a las grandes firmas, sino se aplica a todo tipo de empresa, bien sean públicas o privadas, incluyendo las pymes y las cooperativas. Además de 
permitir la mejora en cuanto a imagen de marca de una empresa, esto conlleva a un impacto real en el valor de la empresa. (p. 79)

En su libro sobre ingeniería de métodos, en donde analiza la variable tiempo, Palacios (2009) señala que:

Las razones que hacen necesario tener estimaciones de tiempo son:

- Las compañías deben cotizar un precio competitivo.

- Para hacer una oferta se debe estimar el tiempo y costo de manufactura.

- Establecer un programa de fabricación.

- Evitar tiempos ociosos de máquinas y operarios.

- Cumplir las fechas de embarque a los clientes.

- Planear la llegada de las materias primas.

- Realizar mantenimiento de equipos, instalaciones, orden y aseo de las plantas.

- Predecir las necesidades de equipo y mano de obra o sea las horas-hombre y horas-máquina. (p. 182)

\section{DISEÑO}

Identificar los datos de entrada, escoger los datos no confiables y eliminarlos, para lograr datos de calidad

Planificación de la producción es la variable independiente.
La planificación de la MOD es la variable dependiente y el resultado esperado.

Estándar de producción expresa la cantidad de horas-hombre $(\mathrm{HH})$ que se requiere para producir una unidad agregada.

En 2013, Bello afirmó que, para poder determinar el factor de producción relacionado con la mano de obra, es necesario determinar el tiempo estándar para cada operación, procedimiento, proceso o actividad. Para explicarlo, Bello desarrolló el procedimiento del método de observación para determinar el tiempo estándar aplicado a una operación de empacado de cajas.

Eficiencia de la MOD representa la relación de la mano de obra directa real frente al estándar.

Distribución de la MOD expresa cómo las horas directas son utilizadas en el proceso de producción: labor directa, labor indirecta y labor no productiva.

\section{Programación del modelo}

Las especificaciones de su funcionalidad.

Modelado del operador del sistema de producción de planificación de la MOD.

En la figura 1 se puede observar los insumos que se requiere para determinar la cantidad de mano de obra directa necesaria para un plan de producción.

La identificación de las variables y parámetros para el modelo de planificación de la MOD permite calcular el número de personas que se necesita para un determinado plan de producción. Ello se puede observar en la tabla 1.

\begin{tabular}{|l|}
\hline \multicolumn{1}{|c|}{ Ingresos } \\
\hline -Plan de producción \\
- Horas estándares \\
- Eficiencia \\
- Horas esperadas \\
-Distribución de la MOD \\
\end{tabular}

\begin{tabular}{|c|}
\hline \multicolumn{1}{|c|}{ Proceso } \\
\hline$\cdot$ N. $^{\circ}$ de días efectivos \\
$\cdot$ N. ${ }^{\circ}$ de turnos \\
$\cdot$ Horas x turno \\
$\cdot$ Horas x operario x período \\
$\cdot$ Costo de HH \\
$\cdot$ Modelo de cálculo de la \\
MOD \\
\hline
\end{tabular}

\begin{tabular}{|c|}
\hline Resultados \\
\hline$\cdot$ N. ${ }^{\circ}$ de operarios a \\
contratar y despedir $^{\circ}$ de horas ociosas \\
$\cdot$ N. ${ }^{\circ}$ de horas extra \\
\\
\hline
\end{tabular}

Figura 1. Planificación de la MOD.

Fuente: elaboración propia. 
Tabla 1. Cálculo de la mano de obra directa.

\begin{tabular}{|c|c|c|}
\hline Signo & Descripción & Fórmula \\
\hline M & Estándar de la MOD & HES $=P(x)^{\star} \mu$ \\
\hline HES & HH estándares & \multirow{4}{*}{ HEE $=\frac{P(x)^{\star} \mu}{e}$} \\
\hline e & Eficiencia de la MOD & \\
\hline HEE & $\mathrm{HH}$ esperadas & \\
\hline LD & $\mathrm{HH}$ directa de la MOD & \\
\hline LI & $\mathrm{HH}$ indirecta de la MOD & $\mathrm{LI}=\mathrm{LI}{ }^{*} \mathrm{TMOD}$ \\
\hline NP & HH no productivos & $N P=N P^{*} T M O D$ \\
\hline TMOD & Total HH de la MOD & \multirow{2}{*}{ TMOD $=\frac{\text { HEE }}{\text { LD }}$} \\
\hline HOP & Horas por operario por periodo & \\
\hline NOPP & Número de operarios por periodo & $\mathrm{HOP}=\mathbf{d}^{*} \boldsymbol{\mu}$ \\
\hline & & $\mathrm{NOPP}=\frac{\mathrm{TMOD}}{\mathrm{HOP}}$ \\
\hline
\end{tabular}

Fuente: elaboración propia.

El objetivo de la planificación de los recursos, en este caso de mano de obra directa, debe estar focalizado en mejorar los procesos e equilibrar el uso de los recursos, tal como menciona Cuatrecasas (2012) en su estudio:

La adecuada organización y la racionalización de los procesos, junto a la utilización de tecnologías avanzadas, nos llevará a esta optimización de la productividad. Esta organización y racionalización alcanzará por supuesto a la clase y calidad de factores productivos utilizados. Dentro de la mencionada racionalización, y en especial en lo referente a la utilización de factores productivos, es importante tener en cuenta el «equilibrio de participación» de los mismos, es decir, las cantidades relativas utilizadas de cada uno. Ya vimos que el óptimo económico se basa en combinar adecuadamente tales cantidades, y en lo que a los aspectos prácticos hace referencia, creemos que es de la mayor importancia el equilibrio: bienes de capital / mano de obra y la correcta cualificación de ésta. (p. 725)

\section{Pruebas}

Luego de aprobado el plan de producción, se simularon los cuatro escenarios:

1. Cuando se cubre la demanda con los trabajadores fijos o nombrados.

2. Cuando la demanda trimestral es mayor al promedio, pero menor que su capacidad.

a. Planificar, contratando y despidiendo. b. Planificar, contratando y fijando horas extra.

c. Planificar, con trabajadores fijos y subcontratar los faltantes.

El plan de producción se calcula en consideración a las necesidades del mercado por abastecer, las políticas de producción, de inventarios, así como la capacidad y los registros de estos mismos (ver tabla 3).

Estándar=20,25 HH/1000 UND; eficiencia=0,80; distribución de la mano de obra directa: labor directa 0,85 ; labor indirecta 0,10 ; labor no productiva 0,05 .

A continuación, se presentan dos escenarios:

a. Cuando se cubre la demanda con los trabajadores fijos o nombrados (ver tabla 3 ).

Se inicia el desarrollo del plan de producción, como puede verse en la tabla 2.

Generalmente, se trata de productos que tienen una producción cuyas desviaciones, bajas o controladas, poseen un personal fijo para la producción y, en épocas de alta, son cubiertas por horas extra, lo cual asegura que las desviaciones de calidad sean mínimas, lo que no sucede cuando se recurre a subcontratar la mano de obra o la producción, donde las desviaciones de calidad son mayores al $10 \%$.

En este tipo de escenario, la toma de decisiones está orientada a mejorar siempre el servicio al cliente interno y externo (ver tabla 5).

b. Cuando la demanda trimestral es mayor al promedio, pero menor que su capacidad de producción (ver tabla 4).

La empresa formula un juego de planes de producción que pueden ser más de uno, el cual es evaluado a nivel de costos y seleccionado. También soporta las necesidades del mercado y las exigencias internas que están dadas por las exigencias a cumplir con las políticas establecidas por las gerencias y el mínimo de inversión.

Para el cálculo de la cantidad de mano de obra directa, puede verse la tabla 5 .

En su libro publicado en 2013, Bello menciona que «el tiempo total por la unidad de producción es la base para el cálculo de: recurso humano, maquinaria, horas-recurso humano, horas-máquinas y para obtener cualquier información relacionada con el proceso productivo» (p. 99). 
Tabla 2. Plan de producción 1.

\begin{tabular}{|c|c|c|c|c|c|c|}
\hline & $\begin{array}{l}\text { Política de } \\
\text { inventario }\end{array}$ & 30 & $\begin{array}{c}\text { Unidades } \mathrm{x} \\
\text { hora }\end{array}$ & 396 & Lote & 2000 \\
\hline Horas extra & $<=$ & $0 \%$ & $\mathrm{H} \times$ turno & 9,60 & & \\
\hline Trimestres & 0 & I & II & III & IV & TOTAL \\
\hline Días efectivos & & 62 & 63 & 62 & 61 & 248 \\
\hline Capacidad & & 234000 & 238000 & 234000 & 230000 & 936000 \\
\hline VENTA & & 100500 & 102500 & 124000 & 99500 & 426500 \\
\hline Órdenes proceso & & 4000 & & & & 4000 \\
\hline Producción & & 122000 & 114000 & 112000 & 104000 & 452000 \\
\hline Inventario proyectado & 24000 & 49500 & 61000 & 49000 & 53500 & 213000 \\
\hline \multicolumn{2}{|l|}{ Días de inventario } & 15 & 30 & 31 & 30 & \\
\hline \multicolumn{2}{|l|}{ Capacidad utilizada } & $52 \%$ & $48 \%$ & $48 \%$ & $45 \%$ & \\
\hline
\end{tabular}

Fuente: elaboración propia.

Tabla 3. Cálculo de mano de obra directa 1.

\begin{tabular}{|c|c|c|c|c|c|}
\hline Estándar de la MOD & 20,25 & & & & \\
\hline HH Estándares & & 2471 & 2309 & 2268 & 2106 \\
\hline Eficiencia de la MOD & 0,80 & & & & \\
\hline HH esperadas & & 3088 & 2886 & 2835 & 2633 \\
\hline \multicolumn{6}{|l|}{ Distribución de la MOD } \\
\hline $\mathrm{HH}$ directa & 0,85 & 3088 & 2886 & 2835 & 2633 \\
\hline $\mathrm{HH}$ indirecta & 0,10 & & & & \\
\hline $\mathrm{HH}$ no productiva & 0,05 & & & & \\
\hline Total de la MOD $(\mathrm{HH})$ & & 3633 & 3395 & 3335 & 3097 \\
\hline Horas $\mathrm{x}$ turno & 9,60 & 595,20 & 604,80 & 595,20 & 585,60 \\
\hline Operario $\mathrm{x}$ trim & & 6,10 & 5,61 & 5,60 & 5,29 \\
\hline Operarios fijos & & 6 & 6 & 6 & 6 \\
\hline MOD normal & & 6 & 6 & 6 & 6 \\
\hline Horas ociosas & & 0 & 39 & 39 & 69 \\
\hline Horas extra & & 62 & 0 & 0 & 0 \\
\hline MOD normal & & 35712 & 36288 & 35712 & 35136 \\
\hline Horas extra & & 928 & 0 & 0 & 0 \\
\hline
\end{tabular}

Fuente: elaboración propia.

Tabla 4. Plan de producción 2.

\begin{tabular}{|c|c|c|c|c|c|c|}
\hline & Política de inventario & 30 & Unid. $\mathbf{x}$ hora & 475 & Lote & 1000 \\
\hline Horas extra & $<=$ & $0 \%$ & $\mathrm{H} \times$ turno & 9,60 & & \\
\hline Periodos & 0 & 1 & II & III & IV & TOTAL \\
\hline Días & & 62 & 63 & 62 & 61 & 248 \\
\hline Capacidad & & 282000 & 286000 & 282000 & 278000 & 1128000 \\
\hline VENTA & & 201000 & 205000 & 268000 & 280000 & 954000 \\
\hline Órdenes proceso & & 4000 & & & & 4000 \\
\hline PRODUCCIÓN & & 271000 & 237000 & 276000 & 258000 & 1042000 \\
\hline Inventario Py & 24,000 & 98000 & 130000 & 138000 & 116000 & 482000 \\
\hline \multicolumn{2}{|l|}{ Días de inventario } & 7 & 30 & 30 & 30 & \\
\hline \multicolumn{2}{|l|}{ Capacidad utilizada } & $96 \%$ & $83 \%$ & $98 \%$ & $93 \%$ & \\
\hline
\end{tabular}

Fuente: elaboración propia. 
Tabla 5. Cálculo de la mano de obra directa 2.

\begin{tabular}{|c|c|c|c|c|c|}
\hline Estándar de la MOD & 20,25 & $I$ & II & III & IV \\
\hline HH estándares & & 5488 & 4799 & 5589 & 5225 \\
\hline Eficiencia de la MOD & 0,80 & & & & \\
\hline HH esperadas & & 6860 & 5999 & 6986 & 6531 \\
\hline \multicolumn{6}{|l|}{ Distribución MOD } \\
\hline $\mathrm{HH}$ directa & 0,85 & 6860 & 5999 & 6986 & 6531 \\
\hline $\mathrm{HH}$ indirecta & 0,10 & & & & \\
\hline $\mathrm{HH}$ no productiva & 0,05 & & & & \\
\hline Total HH de la MOD & & 8070 & 7058 & 8219 & 7683 \\
\hline Horas $\mathrm{x}$ turno & 9,60 & 595,20 & 604,80 & 595,20 & 585,60 \\
\hline Operario $\mathrm{x}$ trim & & 13,56 & 11,67 & 13,81 & 13,12 \\
\hline Operarios fijos & & 6 & 6 & 6 & 6 \\
\hline
\end{tabular}

Fuente: elaboración propia.

Existen dos variables que afectan la planificación de la MOD: la eficiencia, que ajusta el estándar frente a su ejecución, y la distribución, que se compone en:

Labor directa: son las horas que agregan o transforman a los materiales hasta convertirlos en producto terminado.

Labor indirecta: son las horas que apoyan a que la producción alcance los objetivos de calidad y eficiencia. Por ejemplo, la limpieza sin orden de producción, capacitación, etc.

Labor no productiva: son horas que no agregan valor al producto, al proceso, ni apoya a la producción.

Una planificación sin las consideraciones de estas dos variables convierte al sistema de planificación en un sistema "nervioso», porque durante su ejecución el planeador maestro va a tener que tomar decisiones, las cuales varían entre las horas extra o contratar personal a última hora, con las consecuencias de mermar la productividad.

La mano de obra demanda un tratamiento especial por ser un recurso que no forma parte del producto, pero el cumplimiento de su pago es importante para los resultados. Este punto es resaltado por Polo (2017) cuando hace el siguiente análisis:

El costo de la mano de obra son los salarios, prestaciones y demás pagos que se realizan a los operarios y por los operarios y demás trabajadores que laboran en producción, cuya incidencia sobre la producción, ya sea directa o indirecta, es notoria desde todo punto de vista. A diferencia de los materiales, la mano de obra no queda representada físicamente en el producto pero sí su efecto; de ahí que su naturaleza y tratamiento sea por lo tanto diferente a los materiales utilizados en la producción. (p. 84)

\section{Evaluación de la mejor alternativa}

Una vez calculada la cantidad de mano de obra directa, se evalúan alternativas, tal como se observa en las siguientes tablas (6-9), donde se analiza la forma de cómo cubrir este recurso crítico.

De acuerdo con los resultados, a nivel de costos se debería seleccionar la segunda alternativa, que resulta ser la mejor opción para la organización (ver tabla 10).

\section{El método Kaizen en la planificación de la MOD}

La mejora continua debe ser aplicada en toda la cadena de suministros. Lefcovich (2009) aborda ese asunto cuando desarrolla el siguiente análisis:

Reducir a su mínima expresión las actividades irrelevantes en cuanto a la generación de valor añadido para los clientes externos, y reducir al mismo tiempo los niveles de fallas y errores, permitirá generar mayores valores agregados al menor coste posible. Ello es factible eliminando de manera progresiva y sistemática los desperdicios y despilfarros producidos por las diversas actividades $y$ procesos de la empresa. (p. 11)

El proceso de mejora continua en la empresa gestiona un plan maestro de proyectos, donde se ubican los que corresponden al proceso de producción; por ejemplo, la mejora del ciclo de producción, 
Tabla 6. Planificar la mano de obra directa, contratando y despidiendo.

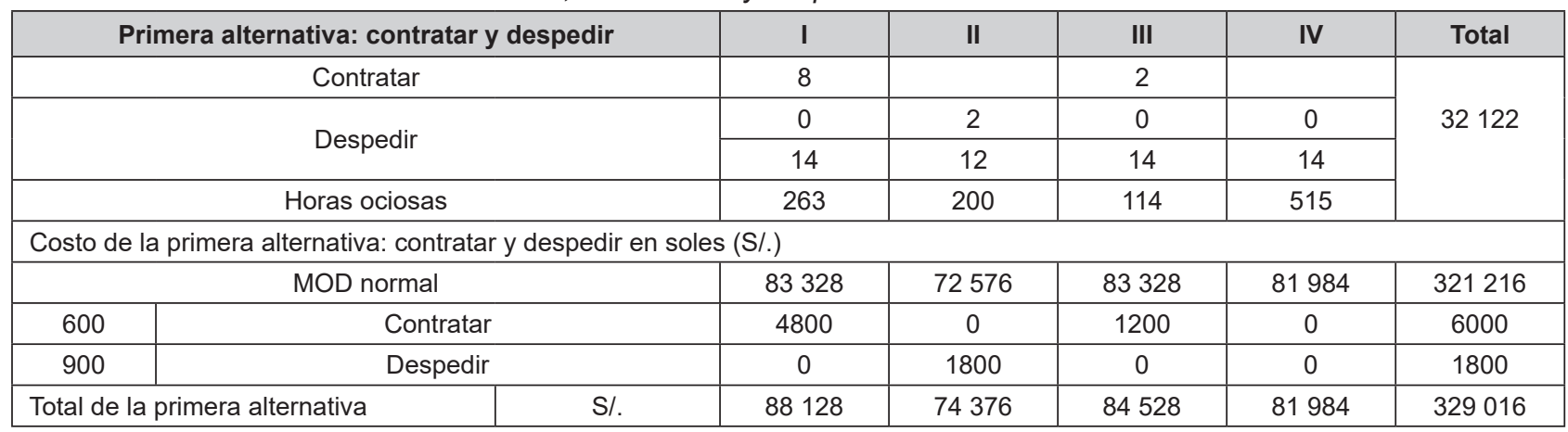

Fuente: elaboración propia.

Tabla 7. Planificar la mano de obra directa, contratando y fijando horas extra.

\begin{tabular}{|c|c|c|c|c|c|}
\hline Segunda alternativa: contratar, despedir y horas extra & I & II & III & IV & Total \\
\hline Contratar & 7 & & 2 & & \multirow[b]{3}{*}{30950} \\
\hline \multirow{2}{*}{ Despedir } & 0 & 2 & & 1 & \\
\hline & 13 & 11 & 13 & 12 & \\
\hline Horas extra & 333 & 405 & 482 & 656 & 1875 \\
\hline \multicolumn{6}{|l|}{ Costo de la segunda alternativa: contratar, despedir y horas extra } \\
\hline MOD normal & 77376 & 66528 & 77376 & 70272 & \\
\hline Horas extra & 4989 & 6074 & 7223 & 9838 & \\
\hline Total de la segunda alternativa & 82365 & 72602 & 84599 & 80110 & 319676 \\
\hline
\end{tabular}

Fuente: elaboración propia.

Tabla 8. Planificar la mano de obra directa con los trabajadores fijos y subcontratar.

\begin{tabular}{|c|c|c|c|c|c|c|c|}
\hline \multicolumn{3}{|c|}{ Tercera alternativa: subcontratar } & 1 & II & III & IV & Total \\
\hline \multirow{3}{*}{ Producción } & \multicolumn{2}{|c|}{ MOD normal } & 119922 & 121856 & 119922 & 117988 & 479687 \\
\hline & \multicolumn{2}{|c|}{ Subcontratar } & 151078 & 115144 & 156078 & 140012 & 562313 \\
\hline & & & 271000 & 237000 & 276000 & 258000 & 1042000 \\
\hline \multicolumn{8}{|c|}{ Costo de tercera alternativa: subcontratar en soles (S/.) } \\
\hline \multirow{2}{*}{$\begin{array}{l}\text { MOD normal } \\
0,50\end{array}$} & & & 36426 & 37014 & 36426 & 35839 & 145705 \\
\hline & Subcontra & & 75539 & 57572 & 78039 & 70006 & 281156 \\
\hline \multicolumn{2}{|c|}{ Total de la tercera alternativa } & $\mathrm{S} /$. & 111965 & 94586 & 114465 & 105845 & 426861 \\
\hline
\end{tabular}

Fuente: elaboración propia.

Tabla 9. Resumen de las alternativas en soles (S/.).

\begin{tabular}{|l|l|}
\hline \multicolumn{2}{|c|}{ Resumen de la valorización de cada alternativa (S/.) } \\
\hline Primera alternativa: contratar y despedir & 329016 \\
\hline Segunda alternativa: contratar y horas extra & 319676 \\
\hline Tercera alternativa: subcontratar & 426861 \\
\hline
\end{tabular}

Fuente: elaboración propia.

que permitirá una reducción de las $\mathrm{HH}$ x 1000 Und. para el III trimestre en la línea de producción seleccionada en este estudio de investigación. En ese sentido, «Mano de Obra Calificada: Se debe contar con personal calificado para evitar fallas y reprocesos, evitando tiempos improductivos» (Álvarez et al., 2004, p. 7).

Aplicado al módulo de planificación de la MOD (tabla 11), los valores de los demás parámetros se mantienen; entonces se obtendrían los resultados que se observan en la tabla10.

Dentro del plan de producción no se proyecta la mejora, por lo tanto, el estándar utilizado permanece inalterable desde el primer hasta el cuarto trimestre:

El proceso de mejora continua demanda una inversión por parte de la gerencia como líder, la cual debe de obtenerse de los procesos mejorados. Adi- 
cionalmente, debe generarse ahorros, los cuales hacen competitiva a la organización, tal como refiere Fernández (2009):

La aplicación de la metodología de mejora exige determinadas inversiones. Es posible y deseable justificar dichas inversiones en términos económicos a través de los ahorros e incrementos de productividad que se producirán por la reducción del ciclo de fabricación [...]. El verdadero progreso en la empresa solo se ha logrado cuando el ejecutivo de más alta jerarquía decide que él personalmente liderará el cambio. (p. 5)

Se proyecta una reducción de $\mathrm{HH}$ en los trimestres III y IV (tabla 12).

Tabla 10. Plan de producción 3.

\begin{tabular}{|c|c|c|c|c|c|c|}
\hline & Política de inventario & 30 & Und $\mathrm{x}$ hora & 475 & Lote & 1000 \\
\hline Horas extra & $<=$ & $0 \%$ & $\mathrm{H} \times$ turno & 9,60 & & \\
\hline Periodos & 0 & 1 & II & III & IV & Total \\
\hline Días & & 62 & 63 & 62 & 61 & 248 \\
\hline Capacidad & & 282000 & 286000 & 282000 & 278000 & 1128000 \\
\hline Venta & & 201000 & 205000 & 268000 & 280000 & 954000 \\
\hline Órdenes proceso & & 4000 & & & & 4000 \\
\hline Producción & & 271000 & 237000 & 276000 & 258000 & 1042000 \\
\hline Inventario Py & 24000 & 98000 & 130000 & 138000 & 116000 & 482000 \\
\hline \multicolumn{2}{|l|}{ Días de inventario } & 7 & 30 & 30 & 30 & \\
\hline \multicolumn{2}{|l|}{ Capacidad utilizada } & $96 \%$ & $83 \%$ & $98 \%$ & $93 \%$ & \\
\hline
\end{tabular}

Fuente: elaboración propia.

Tabla 11. La planificación de la mano de obra directa antes de la mejora.

\begin{tabular}{|l|c|c|c|c|c|}
\hline \multicolumn{1}{|c|}{ Estándar de la MOD } & $\mathbf{2 0 , 2 5}$ & I & II & III & IV \\
\hline HH estándares & & 5488 & 4799 & 5589 & 5225 \\
\hline Eficiencia de la MOD & 0,80 & & & & 698 \\
\hline HH esperadas & & 6860 & 5999 & & \\
\hline Distribución de la MOD & & & & & 6531 \\
\hline Labor directa & 0,85 & 6860 & 5999 & 6986 & \\
\hline Labor indirecta & 0,10 & & & & \\
\hline Labor no productiva & 0,05 & & & & \\
\hline Total HH de la MOD & & 8070 & 7058 & 8219 & 7683 \\
\hline Horas x turno & 9,60 & 595,20 & 604,80 & 595,20 & 585,60 \\
\hline Horas x operario x tri & & 13,56 & 11,67 & 13,81 & 13,12 \\
\hline Operarios fijos & & 6 & 6 & 6 & 6 \\
\hline
\end{tabular}

Fuente: elaboración propia.

Tabla 12. Planificación de la mano de obra directa después de la mejora.

\begin{tabular}{|c|c|c|c|c|c|}
\hline Estándar de la MOD & 20,25 & I & II & III & IV \\
\hline Estándar mejorado de la MOD & 16,20 & & & & \\
\hline HH estándares & & 5488 & 4799 & 4471 & 4180 \\
\hline Eficiencia de la MOD & 0,80 & & & & \\
\hline HH esperadas & & 6860 & 5999 & 5589 & 5225 \\
\hline \multicolumn{6}{|l|}{ Distribución de la MOD } \\
\hline Labor directa & 0,85 & 6860 & 5999 & 5589 & 5225 \\
\hline Labor indirecta & 0,10 & & & & \\
\hline Labor no productive & 0,05 & & & & \\
\hline Total HH de la MOD & & 8070 & 7058 & 6575 & 6146 \\
\hline Horas $x$ turno & 9,60 & 595,20 & 604,80 & 595,20 & 585,60 \\
\hline Horas $\mathrm{x}$ operario $\mathrm{x}$ tri & & 13,56 & 11,67 & 11,05 & 10,50 \\
\hline Operarios fijos & & 6 & 6 & 6 & 6 \\
\hline
\end{tabular}

Fuente: elaboración propia. 


$\begin{array}{lccc} & \text { III } & \text { IV } & \\ \text { Sin mejora } & 8219 & 7683 & \\ \text { Con mejora } & 6575 & 6146 & \\ \text { Total ahorro } & 1644 & 1537=3181 \mathrm{HH}\end{array}$

García (2008) desarrolla un análisis sobre los recursos y, especialmente, sobre la mano de obra, a la que considera de gran relevancia:

El hombre es y seguirá siendo el recurso más valioso de la empresa, aunque no sea de su propiedad [...]. Por todo ello, la empresa no debe de considerar sólo el contrato colectivo de trabajo, sino también responder a las necesidades y esperanzas de los trabajadores y empleados, para contar con su máximo esfuerzo, ya que ellos aportan talento, eficacia y experiencia e invierten los mejores años de su vida en el éxito de la empresa. (pp. 75-76)

La empresa compartió los beneficios alcanzados con los trabajadores. Así, el $10 \%$ de las ganancias logradas a través de las mejoras implementadas, en el periodo de un año, se invirtieron en los trabajadores y empleados que participaron en los diferentes proyectos de mejora. Este reconocimiento es realizado mediante una ceremonia, y cada trabajador es reconocido por escrito, además de entregarles un bien de acuerdo a su aporte.

\section{RESULTADOS}

El modelo de planificación de la MOD es el resultado de la combinación de conocimientos de ingeniería y la experiencia en plantas farmacéuticas, desarrollando planes de producción y diferentes recursos, específicamente el recurso crítico de la mano de obra directa. Por lo tanto, las variables seleccionadas para el modelo de planificación de la MOD permiten ajustar los estándares y logran proyectar una cantidad del recurso crítico al que se le denominará: $\mathrm{HH}$ esperado, por ser lo más cercano a la realidad. El modelo matemático utilizado en la planificación de la MOD permite combinar las variables y parámetros seleccionados; también determina las necesidades del recurso crítico de la mano de obra directa en cantidades muy cercanas a lo real.

En el diseño del modelo de planificación de la MOD, se ha aplicado el método Kaizen (mejora continua), y se proyecta un ahorro del recurso crítico de 3181 $\mathrm{HH}$ frente a la planificación de la mano de obra directa sin mejora.

\section{DISCUSIÓN}

Si el modelo de planificación de la MOD no considera los parámetros eficiencia y distribución de la MOD, como muestran los resultados, las diferencias en un año alcanzarían la cantidad de -4306 $\mathrm{HH}$, con una desviación del $-20,41 \%$. Esto ocasionaría contrataciones de la MOD urgentes, retrasos en la producción, horas extra, afectaría el nivel de servicio al cliente e incrementaría los costos de las operaciones.

La aplicación de la planificación de la MOD demanda a las organizaciones un nivel importante de conocimiento sobre ingeniería industrial y de sus procesos empresariales en la cadena de valor, además de tener determinados conceptos sobre ingeniería industrial, como los desarrollados en la presente investigación.

Asimismo, puede aplicarse el método Kaizen en la planificación de la mano de obra directa, pues es necesario contar con un maestro de proyectos de mejora.

\section{CONCLUSIONES}

- Las variables y parámetros seleccionados para el modelo de PMOD son los correctos para la realidad de la organización, porque permiten ajustar los estándares y proyectar una cantidad del recurso crítico al que se denominará como $\mathrm{HH}$ esperado.

- El modelo matemático utilizado en la PMOD logra una buena combinación de las variables, los parámetros y la ejecución de las operaciones definidas, y así determinar las necesidades del recurso crítico: mano de obra directa en cantidades muy cercanas a lo real.

\section{AGRADECIMIENTOS}

Agradecemos a los docentes y a los gerentes de empresas farmacéuticas por sus comentarios y consejos, entre los cuales se menciona al Ing. Estivene Welsch Romainville y al Dr. Alberto Valle Vega.

\section{REFERENCIAS BIBLIOGRÁFICAS}

[1] Álvarez, J.; Inche, J. y Salvador, G. (2004). Programación de operaciones mediante la teoría de restricciones. Industrial Data, 7(1), 12-19.

[2] Arnoletto, E. J. (2007). Administración de la producción como ventaja competitiva. 
Recuperado de http://www.adizesca.com/site/ assets/g-administracion_de_la_produccion_ como_ventaja_competitiva-ea.pdf.

[3] Bello, C. (2013). Producción y operaciones aplicadas a las pymes. Bogotá, Colombia: Ecoe Ediciones.

[4] Cuatrecasas, L. (2012). Gestión económica de la producción. Madrid, España: Ediciones Díaz de Santos.

[5] Fernández, M. (2009). La mejora continua, una necesidad de estos tiempos. Córdoba, España: El Cid Editor.

[6] Fernández, R. (2013). La mejora de la productividad en la pequeña y mediana empresa. Alicante, España: Editorial Club Universitario.

[7] García, J. (2008). Contabilidad de costos. México, D. F., México: McGraw-Hill / Interamericana Editores.

[8] Heizer, J. y Render, B. (2008). Dirección de la producción y de operaciones. Decisiones tácticas. Madrid, España: Pearson Prentice Hall.
[9] Joseph, N. (2019). Uncertainty management of supply chain manufacturing cost using genetic algorithm. International Journal of Applied Engineering Research, 14(3), 678-683.

[10] Lefcovich, M. (2009). Sistema de mejora continua integral. Córdoba, España: El Cid Editor.

[11] Núñez, A.; Guitart, L. y Baraza, X. (2014). Dirección de operaciones. Barcelona, España: Editorial UOC.

[12] Pacoricuna, E. y Mejía, M. (2015). Mejoras en la programación de la producción de una empresa farmacéutica. Industrial Data, 18(2), 27-35.

[13] Palacios, L. (2009). Ingeniería de métodos. Movimientos y tiempos. Bogotá, Colombia: Ecoe Ediciones.

[14] Peña, C. (2017). Planificación de ventas y operaciones S\&OP en 14 claves. Granada, España: Marge Books.

[15] Polo, B. (2013). Contabilidad de costos en la alta gerencia: teórico-práctico. Bogotá, Colombia: Grupo Editorial Nueva Legislación S. A. S. 


\section{Designing a Direct Labor Planning Model for production management of pharmaceutical companies}

Gustavo Raúl QuisPe Canales ${ }^{1}$

\section{ABSTRACT}

In the pharmaceutical industry, production planning responds to changes in customer demand and internal company processes. Processes in this industry are not value-additive, although the industry does present opportunities for improvement, such as reducing overtime, which occurs more than $30 \%$ of the time; reducing production rescheduling, which reaches $40 \%$; and improving level of customer service for state institutions, who represent $70 \%$ of purchase orders. These situations need to be improved within the company; and as a result, a sales and operations model that includes direct labor planning is designed. This model should enable a reduction of overtime and calculation of the amount of direct labor taking into account efficiency level. In practice, this is the expected outcome; it is achieved via the decisions made by the operations executive.

Keywords: Production plan; direct workforce planning; capacity of direct labor; critical resource; Kaizen method.

\section{INTRODUCTION}

Regarding the definition of the Sales and Operations Planning (S\&OP) model, Núñez et al. (2014) state as follows:

El documento que recoge este nivel de planificación es el denominado plan agregado de producción, en cuyo diseño se tratará de igualar, siempre que sea posible, la tasa de producción a las previsiones de demanda. Sin embargo, dado que esta última suele no ser constante y regular a lo largo del tiempo, el planificador deberá decidir qué medidas de ajuste se deberán tomar para intentar igualarlas. [The document that captures this level of planning is the so-called aggregate production plan, whose design will seek to match, whenever possible, the rate of production with forecasted demand. However, since the latter is often not constant and regular over time, the planner will need to decide what adjustment measures should be taken to try to match them.] (p. 39)

In order to begin designing an aggregate plan, it is important to know the production needs to be met. These should be calculated on the basis of demand forecasts, taking into account the existence of inventories available (reduction of needs) or the existence of outstanding orders left by previous planning (add needs). Thus, the production plan is obtained, the main input in the planning of direct labor (DL).

Accordingly, Arnoletto (2007) comments on the sequence of material requirement planning (mrp) systems, whose main objective is to specify the "combinación óptima de la tasa de producción, del nivel de la fuerza laboral y del inventario disponible" [optimal combination of production rate, workforce level and available inventory]. Production rate is the "número de unidades terminadas por unidad de tiempo (como por hora o por día)" [number of produced units per time unit (such as per hour or per day)]; available inventory "es el saldo del inventario no utilizado traído desde el periodo anterior" [is the balance of the unused inventory of the previous period]; and "el nivel de la fuerza laboral es el número de trabajadores necesario para la producción" [workforce level is the number of workers necessary for production] (p. 142).

Master in Industrial Engineering from the Universidad Ricardo Palma. Currently working as Professor at the Department of Industrial Engineering at the URP. E-mail: gustavo.canales@urp.edu.pe 
Direct labor planning depends on the S\&OP model, which considers which variables affect the result. Peña (2017) addresses it in greater depth in the following analysis:

En definitiva, el S\&OP trata de suavizar el efecto de las fluctuaciones de la demanda del mercado, así como los efectos de los demás cuellos de botella que pueden existir en la organización, y actúa como catalizador para evitar efectos bruscos que repercutan en la cuenta de resultados, y lograr un equilibrio final entre ventas y operaciones. [S\&OP ultimately seeks to soften the effect of fluctuations in market demand, as well as the effects of other bottlenecks that may exist in the organization; it also acts as a catalyst to avoid abrupt effects on results and to achieve a final balance between sales and operations.] (p. 84)

Production processes are further affected by human relations and environmental variables, such as economic, social and political changes, that have an impact on the results of business processes. Joseph (2019) explains it as follows:

Uncertainty can be measured by the frequency of its occurrence, and analyzing the relative contribution and resulting effect on delivery performance. It can quantify whether the impact is minor or major. Many conceptual and mathematical models are proposed and used to manage competitive production/manufacturing under uncertainties. Uncertainties in manufacturing have heterogeneous effects due to the interrelationships between resources and operations.

The lead-time and demand uncertainties are individually and interactively significant determinants of system performance found that as processing time uncertainty increases, simple dispatch heuristics provide performance comparable or superior to that of algorithmically more sophisticated scheduling policies. Again, increasing manufacturing flexibility leads to increased performance and to knob the uncertainty. (p. 679)

\section{PROBLEM}

What would be the impact of designing a Direct Labor Planning Model on the production process of pharmaceutical companies?

Regarding the production system of pharmaceutical companies, Pacoricuna and Mejía (2015) state:
La tipología del sistema de producción corresponde a una producción por lotes con cuatro características principales. En primer lugar, el inicio de la fabricación es posible solo si se tiene la Orden de Trabajo, es decir, existe un pedido que solicita el producto para despacharlo al cliente. En segundo lugar, se cuenta con tres clientes denominados A, B y C. En tercer lugar, la variedad de productos está conformada por 40 diferentes productos; y por último, la demanda en el sector farmacéutico es fluctuante, lo que hace difícil obtener un pronóstico de fabricación en un periodo de tiempo mayor a 6 meses. [The typology of the production system corresponds to a lot production with four main characteristics. First, starting production is only possible if there is a work order, that is, there is an order that requests the product to be shipped to the customer. Second, there are three clients named $A, B$ and $C$. Third, the variety of products is made up of 40 different products; and finally, the demand in the pharmaceutical sector is variable, which makes it difficult to obtain a production forecast for a period longer than 6 months.] (p. 2)

Demand fluctuations in the pharmaceutical market are a frequent variable; therefore, it is an important factor to consider in order to minimize deviations. This implies that direct labor planning must be supervised and monitored, considering the following sequence:

\section{FORECASTING $\rightarrow$ PRODUCTION PLANNING $\rightarrow$ DIRECT LABOR PLANNING}

Where forecasting is the independent variable, production planning is the first-level dependent variable and direct labor planning is the second-level dependent variable.

\section{SPECIFIC PROBLEMS}

What variables and logistic and production parameters are involved in the direct labor planning process in order to obtain alternative plans for its own capacity? How does a Direct Labor Planning Model achieve alternatives to minimize costs?

In their study, Heizer and Render (2008) mention how medium-term planning is integrated with operational planning:

For manufacturers, an aggregate schedule ties a firm's strategic goals to production plans. For service organizations, an aggregate schedule ties strategic goals to workforce schedules. (p. 111) 


\section{METHODOLOGY}

\section{Development of the Direct Labor Planning Model}

The objective is to provide the amount of direct labor personnel the company requires. To do so, it is important to consider the social responsibility mentioned by Fernández (2013)

La responsabilidad social no solo es aplicable a las grandes firmas, sino se aplica a todo tipo de empresa, bien sean públicas o privadas, incluyendo las pymes y las cooperativas. Además de permitir la mejora en cuanto a imagen de marca de una empresa, esto conlleva a un impacto real en el valor de la empresa. [Social responsibility applies not only to large firms, but to all types of enterprises, whether public or private, including SMEs and cooperatives. In addition to enabling an improvement of brand image, this leads to a real impact on the value of the company.] (p. 79)

In his book on method engineering, where the variable time is analyzed, Palacios (2009) indicates that:

Time estimates are needed for the following reasons:

- Companies must quote a competitive price.

- Time and manufacture cost must be estimated to make an offer.

- The establishment of a production program.

- Avoid idle machine and operator times.

- Comply with shipping dates.

- Plan the arrival of raw materials.
- Maintain equipment, facilities, order and plant cleanliness.

- Forecast equipment and labor needs, that is, man-hours and machine-hours. (p. 182)

\section{DESIGN}

Identify input data, select unreliable data and delete them in order to obtain quality data.

Production planning is the independent variable.

Direct labor planning is the dependent variable and the expected result.

Standard of production represents the number of manhours $(\mathrm{MH})$ required to produce an aggregate unit.

In 2013, Bello stated that, in order to determine the labor-related production factor, it is necessary to determine the standard time for each operation, procedure, process or activity. To explain this, Bello developed the observation method to determine the standard time used in a box packing operation.

Direct labor efficiency represents the ratio of real direct labor to the standard.

Direct labor distribution expresses how direct hours are used in the production process: direct labor, indirect labor and non-productive labor.

\section{Model formulation}

Functionality specifications.

Modelling of the direct labor planning production system operator.

Figure 1 shows the inputs required to determine the amount of direct labor required for a production plan.

\begin{tabular}{|l|}
\hline \multicolumn{1}{|c|}{ Inputs } \\
\hline -Production plan \\
-Standard hours \\
-Efficiency \\
-Expected hours \\
-DL distribution \\
\end{tabular}

\begin{tabular}{|l|}
\hline \multicolumn{1}{|c|}{ Process } \\
\hline$\cdot$ No. of working days \\
$\cdot$ No. of shifts \\
$\cdot$ Hours per shift \\
$\cdot$ Hours per worker per \\
period \\
$\cdot$ MH cost \\
$\cdot$ DL calculation model
\end{tabular}

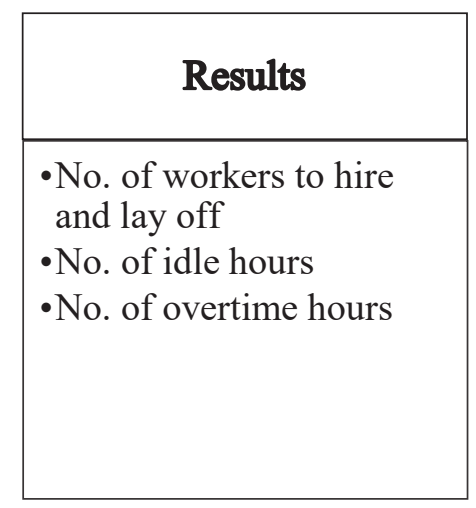

Figure 1. Direct labor planning.

Source: Prepared by the author. 
The identification of variables and parameters for the Direct Labor Planning Model makes it possible to calculate the number of people needed for a given production plan. This can be seen in the following table:

Table 1. Calculation of direct labor.

\begin{tabular}{|c|c|c|}
\hline Symbol & Description & Formula \\
\hline M & DL standard & HES $=P(x)^{*} \mu$ \\
\hline HES & MH standard & \multirow{4}{*}{ HEE $=\frac{P(x)^{*} \mu}{e}$} \\
\hline e & DL efficiency & \\
\hline HEE & Expected MH & \\
\hline LD & $\mathrm{DL}$ direct $\mathrm{MH}$ & \\
\hline LI & $\mathrm{DL}$ indirect $\mathrm{MH}$ & $\mathrm{LI}=\mathrm{LI}{ }^{*} \mathrm{TMOD}$ \\
\hline NP & Non-productive $\mathrm{MH}$ & $N P=N P^{*} T M O D$ \\
\hline TMOD & $\mathrm{DL}$ total $\mathrm{MH}$ & \multirow{2}{*}{ TMOD $=\frac{\text { HEE }}{L D}$} \\
\hline HOP & Hours per worker per period & \\
\hline NOPP & Number of workers per period & $\mathrm{HOP}=\mathbf{d}^{*} \boldsymbol{\mu}$ \\
\hline & & NOPP $=\frac{\text { TMOD }}{\mathrm{HOP}}$ \\
\hline
\end{tabular}

Source: Prepared by the author.

The objective of resource planning, in this case of direct labor, should be focused on improving processes and balancing the use of resources, as stated by Cuatrecasas (2012) in his study:

La adecuada organización y la racionalización de los procesos, junto a la utilización de tecnologías avanzadas, nos llevará a esta optimización de la productividad. Esta organización y racionalización alcanzará por supuesto a la clase y calidad de factores productivos utilizados. Dentro de la mencionada racionalización, y en especial en lo referente a la utilización de factores productivos, es importante tener en cuenta el "equilibrio de participación» de los mismos, es decir, las cantidades relativas utilizadas de cada uno. Ya vimos que el óptimo económico se basa en combinar adecuadamente tales cantidades, y en lo que a los aspectos prácticos hace referencia, creemos que es de la mayor importancia el equilibrio: bienes de capital / mano de obra y la correcta cualificación de ésta. [Proper organization and streamlining of processes, together with the use of advanced technologies, will lead to this optimization of productivity. This organization and streamlining will certainly reach the type and quality of production factors used. In this streamlining, especially regarding the use of production factors, it is important to consider the "balance of participation" of these factors, that is, the quantities used of each factor. We have already observed that the economic optimum is based on a proper combination of such quantities, and as far as practical aspects are concerned, we believe that balance is of the utmost importance: capital goods / labor and its proper qualification] (p. 725)

\section{Testing}

After the production plan was approved, four scenarios were simulated:

1. When demand is met with the permanent or appointed workers.

2. When the quarterly demand is higher than the average, but lower than its production capacity.

a. Planning, hiring and laying off.

b. Planning, hiring and assigning overtime.

c. Planning, with permanent workers and outsourcing those needed.

The production plan is calculated taking into account the needs of the market to be supplied, the production policies, the inventories, as well as the capacity and records (see Table 3 ).

Standard $=20.25 \mathrm{MH} / 1000$ units; efficiency $=0.80$; DL distribution: direct labor 0.85 ; indirect labor 0.10 ; non-productive labor 0.05 .

Two scenarios are presented below:

a. When demand is met with the permanent or appointed workers (see Table 3).

The development of the production plan begins, as can be seen in the table 2 .

These are generally products with a production whose deviations, low or controlled, are conducted by permanent production staff and, in high season, are covered by scheduling overtime. This ensures that quality deviations are minimal, which is not the case when outsourcing labor or production, where quality deviations are greater than $10 \%$.

In this type of scenario, decision-making is always oriented towards improving internal and external customer service (see Table 5).

b. When the quarterly demand is higher than the average, but lower than its production capacity (see Table 4). 
Table 2. Production plan 1.

\begin{tabular}{|c|c|c|c|c|c|c|}
\hline & $\begin{array}{c}\text { Inventory } \\
\text { policy }\end{array}$ & 30 & $\begin{array}{c}\text { Units per } \\
\text { hour }\end{array}$ & 396 & Lot & 2000 \\
\hline Overtime hours & $<=$ & $0 \%$ & $\mathrm{H} \times$ shift & 9,60 & & \\
\hline Quarter & 0 & 1 & II & III & IV & TOTAL \\
\hline Working days & & 62 & 63 & 62 & 61 & 248 \\
\hline Capacity & & 234000 & 238000 & 234000 & 230000 & 936000 \\
\hline SALE & & 100500 & 102500 & 124000 & 99500 & 426500 \\
\hline Process orders & & 4000 & & & & 4000 \\
\hline Production & & 122000 & 114000 & 112000 & 104000 & 452000 \\
\hline Projected inventory & 24000 & 49500 & 61000 & 49000 & 53500 & 213000 \\
\hline \multicolumn{2}{|l|}{ Days in inventory } & 15 & 30 & 31 & 30 & \\
\hline \multicolumn{2}{|l|}{ Used capacity } & $52 \%$ & $48 \%$ & $48 \%$ & $45 \%$ & \\
\hline
\end{tabular}

Source: Prepared by the author.

Table 3. Calculation of direct labor 1.

\begin{tabular}{|c|c|c|c|c|c|}
\hline DL standard & 20.25 & & & & \\
\hline Standard $\mathrm{MH}$ & & 2471 & 2309 & 2268 & 2106 \\
\hline DL efficiency & 0.80 & & & & \\
\hline Expected MH & & 3088 & 2886 & 2835 & 2633 \\
\hline \multicolumn{6}{|l|}{ DL distribution } \\
\hline Direct $\mathrm{MH}$ & 0.85 & 3088 & 2886 & 2835 & 2633 \\
\hline Indirect $\mathrm{MH}$ & 0.10 & & & & \\
\hline Non-productive $\mathrm{MH}$ & 0.05 & & & & \\
\hline Total DL (MH) & & 3633 & 3395 & 3335 & 3097 \\
\hline Hours per shift & 9.60 & 595.20 & 604.80 & 595.20 & 585.60 \\
\hline Workers per quarter & & 6.10 & 5.61 & 5.60 & 5.29 \\
\hline Permanent workers & & 6 & 6 & 6 & 6 \\
\hline Normal DL & & 6 & 6 & 6 & 6 \\
\hline Idle hours & & 0 & 39 & 39 & 69 \\
\hline Overtime hours & & 62 & 0 & 0 & 0 \\
\hline Normal DL & & 35712 & 36288 & 35712 & 35136 \\
\hline Overtime hours & & 928 & 0 & 0 & 0 \\
\hline
\end{tabular}

Source: Prepared by the author.

Table 4. Production plan 2.

\begin{tabular}{|c|c|c|c|c|c|c|}
\hline & Inventory policy & 30 & Units per hour & 475 & Lot & 1000 \\
\hline Overtime hours & $<=$ & $0 \%$ & $\mathrm{H} \times$ shift & 9.60 & & \\
\hline Periods & 0 & 1 & II & III & IV & TOTAL \\
\hline Days & & 62 & 63 & 62 & 61 & 248 \\
\hline Capacity & & 282000 & 286000 & 282000 & 278000 & 1128000 \\
\hline SALE & & 201000 & 205000 & 268000 & 280000 & 954000 \\
\hline Process orders & & 4000 & & & & 4000 \\
\hline PRODUCTION & & 271000 & 237000 & 276000 & 258000 & 1042000 \\
\hline $\begin{array}{l}\text { Projected inven- } \\
\text { tory }\end{array}$ & 24.000 & 98000 & 130000 & 138000 & 116000 & 482000 \\
\hline \multicolumn{2}{|l|}{ Days in inventory } & 7 & 30 & 30 & 30 & \\
\hline \multicolumn{2}{|l|}{ Used capacity } & $96 \%$ & $83 \%$ & $98 \%$ & $93 \%$ & \\
\hline
\end{tabular}

Source: Prepared by the author. 
Table 5. Calculation of direct labor 2 .

\begin{tabular}{|l|c|c|c|c|c|}
\hline DL standard & $\mathbf{2 0 . 2 5}$ & I & II & III & IV \\
\hline Standard MH & & 5488 & 4799 & 5589 & 5225 \\
\hline DL efficiency & 0.80 & & & & 6531 \\
\hline Expected MH & & 6860 & 5999 & 6986 & \\
\hline DL distribution & & & & & 6531 \\
\hline Direct MH & 0.85 & 6860 & 5999 & 6986 & \\
\hline Indirect MH & 0.10 & & & & \\
\hline Non-productive MH & 0.05 & & & & \\
\hline DL total MH & & 8070 & 7058 & 8219 & 7683 \\
\hline Hours per shift & 9.60 & 595.20 & 604.80 & 595.20 & 585.60 \\
\hline Workers per quarter & & 13.56 & 11.67 & 13.81 & 13.12 \\
\hline Permanent workers & & 6 & 6 & 6 & 6 \\
\hline
\end{tabular}

Source: Prepared by the author.

The company formulates a set of production plansthere can be more than one-which is evaluated at cost level and selected. It also supports the needs of the market and the internal requirements determined by the policies established by management, keeping investment to a minimum.

For the calculation of the amount of direct labor, see the following table:

In his book published in 2013, Bello mentions that "el tiempo total por la unidad de producción es la base para el cálculo de: recurso humano, maquinaria, horas-recurso humano, horas-máquinas y para obtener cualquier información relacionada con el proceso productivo" [the total time per production unit is the basis for the calculation of human resources, machinery, man-hours, machine-hours, and for obtaining any information related to the production process] (p. 99).

There are two variables that affect direct labor planning: efficiency, which adjusts the standard to execution, and distribution, which is composed of:

Direct labor: These are the hours that add or transform materials into finished products.

Indirect labor: These are the hours that help production to achieve quality and efficiency objectives. For example, cleaning, training, among others.

Non-productive labor: These are the hours that do not add value to the product or process, nor support production.

Planning without considering these two variables makes the planning system an "uneasy" system, because during its execution the master planner will have to make decisions, which vary between overtime or last-minute recruitment, with the consequences of reducing productivity.

The labor force demands special treatment as it is not part of the product; compliance with payment is important for the results. This point is highlighted by Polo (2017) when he makes the following analysis:

El costo de la mano de obra son los salarios, prestaciones y demás pagos que se realizan a los operarios y por los operarios y demás trabajadores que laboran en producción, cuya incidencia sobre la producción, ya sea directa o indirecta, es notoria desde todo punto de vista. A diferencia de los materiales, la mano de obra no queda representada físicamente en el producto pero sí su efecto; de ahí que su naturaleza y tratamiento sea por lo tanto diferente a los materiales utilizados en la producción. [The cost of labor is the wages, benefits and other payments made to operators and other employees working in production, whose impact on production, whether direct or indirect, is notorious from every point of view. Unlike materials, labor is not physically represented in the product but its impact is; hence its nature and treatment is therefore different from the materials used in production.] (p. 84)

\section{Evaluation of the best alternative}

Once the amount of direct labor has been calculated, alternatives are evaluated, as seen in the following tables (6-9), where the manner how to cover this critical resource is analyzed. 
Table 6. Direct labor planning, hiring and laying off.

\begin{tabular}{|c|c|c|c|c|c|c|}
\hline \multicolumn{2}{|r|}{ First alternative: hire and lay off } & $\mathbf{I}$ & II & III & IV & Total \\
\hline \multicolumn{2}{|r|}{ Hire } & 8 & & 2 & & \multirow{4}{*}{32122} \\
\hline \multirow{2}{*}{\multicolumn{2}{|c|}{ Lay off }} & 0 & 2 & 0 & 0 & \\
\hline & & 14 & 12 & 14 & 14 & \\
\hline \multicolumn{2}{|r|}{ Idle hours } & 263 & 200 & 114 & 515 & \\
\hline \multicolumn{7}{|c|}{ Cost in soles (S/.) of the first alternative: hire and lay off } \\
\hline \multicolumn{2}{|r|}{ Normal DL } & 83328 & 72576 & 83328 & 81984 & 321216 \\
\hline 600 & Hire & 4800 & 0 & 1200 & 0 & 6000 \\
\hline 900 & Lay off & 0 & 1800 & 0 & 0 & 1800 \\
\hline Total of & first alternative & 88128 & 74376 & 84528 & 81984 & 329016 \\
\hline
\end{tabular}

Source: Prepared by the author.

Table 7. Direct labor planning, hiring and assigning overtime.

\begin{tabular}{|c|c|c|c|c|c|}
\hline Second alternative: hire, lay off and overtime hours & $\mathbf{I}$ & II & III & IV & Total \\
\hline Hire & 7 & & 2 & & \multirow[b]{3}{*}{30950} \\
\hline \multirow{2}{*}{ Lay off } & 0 & 2 & & 1 & \\
\hline & 13 & 11 & 13 & 12 & \\
\hline Overtime hours & 333 & 405 & 482 & 656 & 1875 \\
\hline \multicolumn{6}{|l|}{ Cost of the second alternative: hire, lay off and overtime hours } \\
\hline Normal DL & 77376 & 66528 & 77376 & 70272 & \\
\hline Overtime hours & 4989 & 6074 & 7223 & 9838 & \\
\hline Total of the second alternative & 82365 & 72602 & 84599 & 80110 & 319676 \\
\hline
\end{tabular}

Source: Prepared by the author.

Table 8. Direct labor planning with permanent and outsourced workers.

\begin{tabular}{|l|l|c|c|c|c|c|}
\hline \multicolumn{2}{|c|}{ Third alternative: outsourcing } & I & II & III & IV & Total \\
\hline \multirow{3}{*}{ Production } & Normal DL & 119922 & 121856 & 119922 & 117988 & 479687 \\
\cline { 2 - 7 } & Outsourcing & 151078 & 115144 & 156078 & 140012 & 562313 \\
\cline { 2 - 7 } & 271000 & 237000 & 276000 & 258000 & 1042000 \\
\hline \multicolumn{7}{|l|}{ Cost in soles (S/.) of the first third alternative: outsourcing } \\
\hline $\begin{array}{l}\text { Normal DL } \\
0.50\end{array}$ & 36426 & 37014 & 36426 & 35839 & 145705 \\
\hline \multicolumn{2}{|l|}{ Total of the third alternative } & 75539 & 57572 & 78039 & 70006 & 281156 \\
\hline
\end{tabular}

Source: Prepared by the author.

Table 9. Summary of the alternatives in soles (S/.).

\begin{tabular}{|c|c|}
\hline \multicolumn{2}{|c|}{ Summary of the valuation of each alternative (S/.) } \\
\hline First alternative: hire and lay off & 329016 \\
\hline Second alternative: hire and overtime & 319676 \\
\hline Third alternative: outsourcing & 426861 \\
\hline
\end{tabular}

Source: Prepared by the author.

According to the results, considering cost level, the second alternative proves to be the best option for the organization and should be selected (see Table 10).

The Kaizen approach to direct labor planning

Continuous improvement must be applied throughout the supply chain. Lefcovich (2009) addresses this issue in the following analysis:
Reducir a su mínima expresión las actividades irrelevantes en cuanto a la generación de valor añadido para los clientes externos, $y$ reducir al mismo tiempo los niveles de fallas y errores, permitirá generar mayores valores agregados al menor coste posible. Ello es factible eliminando de manera progresiva y sistemática los desperdicios y despilfarros producidos por las diversas actividades $y$ 
procesos de la empresa. [Reducing irrelevant activities to a minimum in terms of generating added value for external customers, as well as reducing failures and errors, will generate greater aggregated values at the lowest possible cost. This can be done by progressively and systematically eliminating misuse and waste produced by the various company activities and processes.] (p. 11)
The continuous improvement process in the company manages an improvement project master plan, where production process plans are found; for example, the improvement of production cycle, which will allow a reduction of $\mathrm{MH} \times 1000$ units for the third quarter in the production line selected in this research study. In this sense, "Mano de Obra Calificada: Se debe contar con personal calificado para evitar fallas y reprocesos, evitando tiempos

Table 10. Production plan 3.

\begin{tabular}{|l|c|c|c|c|c|c|}
\hline & Inventory policy & $\mathbf{3 0}$ & Units per hour & $\mathbf{4 7 5}$ & Lot & $\mathbf{1 0 0 0}$ \\
\hline Overtime hours & $<=$ & $0 \%$ & $\mathrm{H} \times$ shift & 9.60 & & \\
\hline Periods & 0 & $\mathrm{I}$ & $\mathrm{II}$ & $\mathrm{III}$ & $\mathrm{IV}$ & Total \\
\hline Days & & 62 & 63 & 62 & 61 & 248 \\
\hline Capacity & & 282000 & 286000 & 282000 & 278000 & 1128000 \\
\hline Sale & & 201000 & 205000 & 268000 & 280000 & 954000 \\
\hline Process orders & & 4000 & & & 25000 & 1042000 \\
\hline Production & & 271000 & 237000 & 276000 & 258000 \\
\hline Projected inventory & 24000 & 98000 & 130000 & 138000 & 116000 & 482000 \\
\hline Days in inventory & & 7 & 30 & 30 & 30 & \\
\hline Used capacity & & $96 \%$ & $83 \%$ & $98 \%$ & $93 \%$ & \\
\hline
\end{tabular}

Source: Prepared by the author.

Table 11. Direct labor planning before improvement.

\begin{tabular}{|l|c|c|c|c|c|}
\hline \multicolumn{1}{|c|}{ DL standard } & $\mathbf{2 0 . 2 5}$ & I & II & III & IV \\
\hline Standard MH & & 5488 & 4799 & 5589 & 5225 \\
\hline DL efficiency & 0.80 & & & & 6531 \\
\hline Expected MH & & 6860 & 5999 & 6986 & \\
\hline DL distribution & & & & & 6531 \\
\hline Direct labor & 0.85 & 6860 & 5999 & 6986 & \\
\hline Indirect labor & 0.10 & & & & \\
\hline Non-productive labor & 0.05 & & & & \\
\hline DL total MH & & 8070 & 7058 & 8219 & 7683 \\
\hline Hours per shift & 9.60 & 595.20 & 604.80 & 595.20 & 585.60 \\
\hline Hours $x$ worker x quarter & & 13.56 & 11.67 & 13.81 & 13.12 \\
\hline Permanent workers & & 6 & 6 & 6 & 6 \\
\hline
\end{tabular}

Source: Prepared by the author.

Table 12. Direct labor planning after improvement.

\begin{tabular}{|c|c|c|c|c|c|}
\hline DL standard & 20.25 & $I$ & II & III & IV \\
\hline Improved DL standard & 16.20 & & & & \\
\hline Standard $\mathrm{MH}$ & & 5488 & 4799 & 4471 & 4180 \\
\hline DL efficiency & 0.80 & & & & \\
\hline Expected $\mathrm{MH}$ & & 6860 & 5999 & 5589 & 5225 \\
\hline \multicolumn{6}{|l|}{ DL distribution } \\
\hline Direct labor & 0.85 & 6860 & 5999 & 5589 & 5225 \\
\hline Indirect labor & 0.10 & & & & \\
\hline Non-productive labor & 0.05 & & & & \\
\hline $\mathrm{DL}$ total $\mathrm{MH}$ & & 8070 & 7058 & 6575 & 6146 \\
\hline Hours per shift & 9.60 & 595.20 & 604.80 & 595.20 & 585.60 \\
\hline Hours $\mathrm{x}$ worker $\mathrm{x}$ quarter & & 13.56 & 11.67 & 11.05 & 10.50 \\
\hline Permanent workers & & 6 & 6 & 6 & 6 \\
\hline
\end{tabular}

Source: Prepared by the author. 
improductivos" [Skilled labor: qualified personnel must be available to prevent failures and reprocessing, avoiding downtime] (Álvarez et al., 2004, p. 7).

When the Kaizen approach is applied to the Direct Labor Planning Model (Table 11) the values of the other parameters remain unchanged. The results obtained are shown in the following table:

Continuous improvement is not included within the production plan, thus, the standard used remains unchanged from the first to the fourth quarter:

The process of continuous improvement requires an investment by management as the leader, which should be obtained from the improved processes. In addition, savings should be generated, which make the organization competitive, as indicated by Fernández (2009):

La aplicación de la metodología de mejora exige determinadas inversiones. Es posible y deseable justificar dichas inversiones en términos económicos a través de los ahorros e incrementos de productividad que se producirán por la reducción del ciclo de fabricación [...]. El verdadero progreso en la empresa solo se ha logrado cuando el ejecutivo de más alta jerarquía decide que él personalmente liderará el cambio. [The application of the improvement methodology requires certain investments. It is possible and desirable to justify such investments in economic terms through the savings and productivity gains that will result from the reduction of the manufacturing cycle [...]. Real progress in the company has only been made when the top executive decides that he will personally lead the change.] (p. 5)

A reduction of man-hours in the III and IV quarters is projected (Table 12).

$\begin{array}{lcc} & \text { III } & \text { IV } \\ \text { Without improvement } & 8219 & 7683 \\ \text { With improvement } & 6575 & 6146 \\ \text { Total saved } & 1644 & 1537=3181 \mathrm{MH}\end{array}$

García (2008) develops an analysis of resources and, particularly, on labor, which he considers highly relevant:

El hombre es y seguirá siendo el recurso más valioso de la empresa, aunque no sea de su propiedad [...]. Por todo ello, la empresa no debe de considerar sólo el contrato colectivo de trabajo, sino también responder a las necesidades y esperanzas de los trabajadores y empleados, para contar con su máximo esfuerzo, ya que ellos aportan talento, eficacia y experiencia e invierten los mejores años de su vida en el éxito de la empresa. [Man is and will remain the most valuable resource of the company, even if it is not company-owned [...]. Therefore, the company should not only consider the collective labor contract, but also respond to the needs and hopes of the workers and employees, to count on their maximum effort, since they bring talent, efficiency and experience and invest the best years of their lives in the success of the company.] (pp. 75-76)

The company shared benefits obtained with workers. Thus, $10 \%$ of the profits achieved through the improvements implemented, in the period of one year, were invested in the workers and employees who participated in the different improvement projects. This recognition was made via a ceremony, and each worker was recognized in writing, as well as given a gift according to their contribution.

\section{RESULTS}

The Direct Labor Planning Model results from the combination of engineering knowledge, experience of pharmaceutical plants in developing production plans and different resources, specifically the critical resource of direct labor. Therefore, the variables selected for the Direct Labor Planning Model make it possible to adjust the standards and manage to project and successfully forecast the critical resource amount called expected $\mathrm{MH}$, as it is the closest to reality. The mathematical model used in the planning model makes it possible to combine selected variables and parameters; it also determines the need of critical resources of direct labor in quantities very close to the real amount.

The Kaizen approach (continuous improvement) was applied in the designing of the Direct Labor Planning Model, and a critical resource saving of $3181 \mathrm{MH}$ was projected compared to direct labor planning without improvement.

\section{DISCUSSION}

If the Direct Labor Planning Model does not consider efficiency and distribution parameters of direct labor, as shown by the results, the differences in one year would reach $-4306 \mathrm{MH}$, with a deviation of $-20.41 \%$. This would lead to urgent direct labor hi- 
ring, production delays, and overtime; it would also affect the level of customer service and increase operating costs.

The implementation of the Direct Labor Planning Model requires organizations to have a significant level of knowledge of industrial engineering and its business processes in the value chain, including knowledge about specific industrial engineering concepts industrial engineering, such as those developed in this research.

Similarly, the Kaizen method can be applied in planning direct labor, as it is necessary to have an improvement projects master planner.

\section{CONCLUSIONS}

- The variables and parameters selected for the Direct Labor Planning Model were appropriate for the reality of the organization, because they made it possible to adjust the standards and project the amount of a critical resource that will be referred as expected $\mathrm{MH}$.

- The mathematical model used in the Direct Labor Planning Model achieved a good combination of variables, parameters and execution of the defined operations, and thus it was possible to determine the needs of the critical resource: direct labor in quantities very close to the real amount.

\section{ACKNOWLEDGEMENTS}

To the professors and managers of the pharmaceutical companies for their comments and suggestions, including Estivene Welsch Romainville and Dr. Alberto Valle Vega.

\section{REFERENCES}

[1] Álvarez, J.; Inche, J. \& Salvador, G. (2004). Programación de operaciones mediante la teoría de restricciones. Industrial Data, 7(1), 12-19.

[2] Arnoletto, E. J. (2007). Administración de la producción como ventaja competitiva. Retrieved from http://www.adizesca.com/site/ assets/g-administracion_de_la_produccion_ como_ventaja_competitiva-ea.pdf.
[3] Bello, C. (2013). Producción y operaciones aplicadas a las pymes. Bogotá, Colombia: Ecoe Ediciones.

[4] Cuatrecasas, L. (2012). Gestión económica de la producción. Madrid, Spain: Ediciones Díaz de Santos.

[5] Fernández, M. (2009). La mejora continua, una necesidad de estos tiempos. Córdoba, Spain: El Cid Editor.

[6] Fernández, R. (2013). La mejora de la productividad en la pequeña y mediana empresa. Alicante, Spain: Editorial Club Universitario.

[7] García, J. (2008). Contabilidad de costos. México, D. F., Mexico: McGraw-Hill / Interamericana Editores.

[8] Heizer, J. \& Render, B. (2008). Dirección de la producción y de operaciones. Decisiones tácticas. Madrid, Spain: Pearson Prentice Hall.

[9] Joseph, N. (2019). Uncertainty management of supply chain manufacturing cost using genetic algorithm. International Journal of Applied Engineering Research, 14(3), 678-683.

[10] Lefcovich, M. (2009). Sistema de mejora continua integral. Córdoba, Spain: El Cid Editor.

[11] Núñez, A.; Guitart, L. \& Baraza, X. (2014). Dirección de operaciones. Barcelona, Spain: Editorial UOC.

[12] Pacoricuna, E. \& Mejía, M. (2015). Mejoras en la programación de la producción de una empresa farmacéutica. Industrial Data, 18(2), 27-35.

[13] Palacios, L. (2009). Ingeniería de métodos. Movimientos y tiempos. Bogotá, Colombia: Ecoe Ediciones.

[14] Peña, C. (2017). Planificación de ventas y operaciones S\&OP en 14 claves. Granada, Spain: Marge Books.

[15] Polo, B. (2013). Contabilidad de costos en la alta gerencia: teórico-práctico. Bogotá, Colombia: Grupo Editorial Nueva Legislación S. A. S. 